\title{
SPECTRAL MONITORING OF AGN AT THE 6 METER TELESCOPE
}

\author{
N.G. BOCHKAREV \\ Astronomical Society, Sternberg Astronomical Institute, Universitetskij \\ prosp.13, Moscow, 119899, Russia \\ AND \\ A.I. SHA POVALOVA, A.N.BURENKOV, V.V.VLASYUK \\ Special Astrophysical Observatory, N.Arkhyz, 357147. Russia
}

\section{Observations}

To study broad line region (BLR) size and kinematics spectral monitoring of NGC 3516, NGC 4151, NGC 5548, NGC 7469 and 3C390.3 has been performed with the $6 \mathrm{~m}$ telescope of the Special Astrophysical Observatory (N.Arkhyz- North Caucasus) in 1986-1996 . The spectra are obtained in the Nasmyth focus using the TV scanner with a resolution of $3-4 \AA$ and $\mathrm{S} / \mathrm{N}=10-25$ within $4000-5200 \AA \AA$ (1986-94, 500 spectra), and in the prime focus with the spectrograph UAGS (a long-slit mode) + CCD (resolution

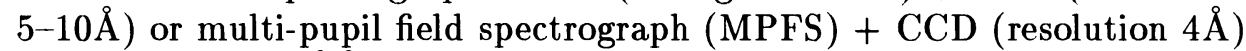
within $4000-7000 \AA \AA$ and $\mathrm{S} / \mathrm{N}=50-100$ (1995-96, 270 spectra).

\section{Results}

NGC 4151 (1986-96): In 1984-1987 the nucleus has passed through a deep minimum of brightness. During the interval 1987-1995, the broad component's brightness has increased by a factor of 8 . During the decade , red and blue wings' variations for Balmer lines were simultaneous, the blue wing keeping brighter than the red one (Shapovalova et.al.,1996). At the same time, the HeII $4686 \AA$ line broad component wings varied quasiperiodically: the dominant wing of the line changed every two years (Bochkarev et.al, 1996). Scrupulous observations of the emission spectrum of the NGC 4151 nucleus during its deep minimum of 1986-1987, with the broad emission lines almost absent, allowed to assume, using the averaged spectra with 
high S/N ratio, the existence of narrow satellites of Balmer lines (Bochkarev et.al.,1991).

NGC 5548 (1988-93): In the frame of the AGN Watch Program (Peterson et al., 1991, 1994; Korista et.al, 1995), $\mathrm{R}$ was determined not only for $H_{\beta}$ but also for high ionization line (CIV $1550 \AA$ ) formation region. It was confirmed that high charge ions emit mostly nearer to the ionizing radiation source than hydrogen, and, moreover, the region dimensions differ several times. FeII broad line formation region was studied (Maoz et al., 1993).

3C390.3 (1995-96): Quasisimultaneous variations of the fluxes in the blue and red wings of $H_{\beta}$ are detected. We have also confirmed the quasiperiodicity for the fluxes ratios of the blue and red wings of $H_{\beta}$. (Shapovalova et.al., 1996). It is shown that radiation in [OIII] $4959,5007 \AA \AA$ emission lines corresponds to the region with $\mathrm{r} \approx 2$ arcsec.

\section{Summary}

The principle results obtained in the past 10 years are as follows:

- the absence of significant radial motion inside the BLR in NGC 4151, 3C390.3 and, possibly, in NGC 5548 has been proved on the basis of numerous high quality data;

- BLR sizes are shown to be about two times smaller in comparison with the parameter assumed before;

- stratification of BLR follows from the observations.

- broad FeII lines behavior is similar to that of the $H_{\beta}$ line;

- it was assumed that Balmer lines in NGC 4151 nucleus have narrow satellites, which yield information on jet-formation region.

- the new narrow emission line parameters (NGC 3516, NGC 4151) and the dimensions of certain narrow emission-line formation regions (3C390.3) were determined.

This research is supported by Russian Foundation for Basic Research grant 97-02-17625 and the Russian Federal Science-Tecknical Program "Astronomy."

\section{References}

Bochkarev N.G., Shapovalova A.I., Zhekov S.A., 1991, Astron.J. 102,1278

Bochkarev N.G., Shapovalova A.I., Nazarova L.S., 1996, Astron.Astrophys.Trans. 10,241 Korista K.T. et.al., 1995, ApJS 97, 285

Maoz D. et.al., 1993, ApJ 404, 576

Peterson B.M. et.al., 1991, ApJ 368,119; 1993, ApJ 425,622

Shapovalova A.I., Burenkov A.N., Bochkarev N.G., 1996, Bul. Spec. Astrophys. Obs. 41,28 . 